\title{
Clases virtuales en el ingreso universitario: la emergencia en la construcción de sentido
}

\section{Virtual classes in university entrance: the emergency in the construction of meaning}

\author{
Almirón, Alejandra \\ https://orcid.org/0000-0001-5146-5738 \\ alealmi83@gmail.com \\ Instituto de Estudios Iniciales | UNAJ | Argentina
}

Cabaña, Lorena

https://orcid.org/0000-0002-8639-1297

lorena.cabana59@gmail.com

Instituto de Estudios Iniciales | UNAJ | Argentina

\section{Bifano, Fernando}

https://orcid.org/0000-0001-5925-5794

fibifano@gmail.com

Instituto de Estudios Iniciales | UNAJ | Argentina

González, Karina

https://orcid.org/0000-0002-7944-2463

karinafiles@yahoo.com.ar

Instituto de Estudios Iniciales | UNAJ | Argentina

\section{RESUMEN}

La coyuntura actual en la que el mundo se encuentra, a partir de la declaración de pandemia por el COVID-19, ha significado -y significa aún- un contexto de gran desafío para la "continuidad pedagógica" en todo el ámbito de la educación. El nivel universitario, lejos se encuentra de estar exento de esta situación. Apoyándose en la autonomía universitaria que lo faculta, ha decidido de diversa manera atender y dar respuesta para garantizar el derecho universal a la educación. En este marco, la Universidad Nacional Arturo Jauretche -en adelante UNAJ-, ha analizado pormenorizadamente, cuáles eran las materias que podían, con las limitaciones del caso, hacer una oferta de clases a través de aulas virtuales.

En esta reseña de investigación en marcha, daremos cuenta del proceso que hemos comenzado a partir de un cuestionario sobre expectativas docentes para las y los profesores de las materias que componen el ciclo inicial de la UNAJ. El procesamiento de los primeros resultados nos ha generado diferentes preguntas acerca de este contexto de virtualidad forzada que nos toca atravesar: ¿cómo afectarán a docentes y estudiantes las condiciones de confinamiento en el hogar y de accesibilidad a las tecnologías digitales para enseñar y aprender en este marco de continuidad pedagógica? ¿En qué medida la falta de experiencia de docentes y estudiantes en entornos virtuales será un obstáculo para la enseñanza y el aprendizaje? ¿En qué consiste una clase virtual en este marco? ¿Cómo definir sus límites espaciotemporales? ¿Cómo se pueden compatibilizar expectativas entre lo que las y los docentes ofrecen para sostener la enseñanza con lo que las y los estudiantes esperan recibir?, son algunas de las muchas preguntas que empiezan a surgir de la dinámica de trabajo. En particular, en esta presentación trataremos de comenzar a delinear los elementos emergentes para elaborar una respuesta en lo referente con la construcción de sentidos en una "clase virtual" a partir de apelar a una posible definición desde la metodología de la teoría fundamentada basada en el análisis de lo que las y los docentes entienden y asumen que es una clase virtual en este contexto.

PALABRAS CLAVE

Continuidad Pedagógica, Virtualidad Forzada, Clases Virtuales, Teoría fundamentada. 
KEY WORDS

Pedagogical Continuity, Forced Virtuality, Virtual Classes, Grounded theory.

\section{ABSTRACT}

The current situation in which the world finds itself, since the declaration of a pandemic by COVID-19, has meant - and still means - a context of great challenge for "pedagogical continuity" in the entire field of education. The university level is far from being exempt from this situation. Relying on the university autonomy that empowers it, it has decided in various ways to attend and respond to guarantee the universal right to education. Within this framework, the National Arturo Jauretche University -after UNAJ-, has analyzed in detail, which were the subjects that could, with the limitations of the case, make an offer of classes through virtual classrooms.

In this review of ongoing research, we will give an account of the process that we have started from a questionnaire on teaching expectations for the teachers of the subjects that make up the initial cycle of UNAJ. The processing of the first results has generated different questions about this context of forced virtuality that we have to go through: how will the conditions of home confinement and accessibility to digital technologies for teaching and learning in this context affect teachers and students? pedagogical continuity framework? To what extent will the lack of experience of teachers and students in virtual environments be an obstacle to teaching and learning? What is a virtual class in this framework? How to define its spatio-temporal limits? How can expectations be reconciled between what teachers offer to sustain teaching with what students expect to receive? These are some of the many questions that are beginning to emerge from the work dynamics. In particular, in this presentation we will try to begin to delineate the emerging elements in order to elaborate an answer regarding the construction of meanings in a "virtual class" starting from appealing to a possible definition from the methodology of grounded theory based on the analysis of what teachers understand and assume is a virtual class in this context. 


\section{PRESENTACIÓN}

La UNAJ está situada en el segundo cordón del conurbano bonaerense y recibe año a año a miles de estudiantes de la zona que suelen ser primera generación que concurren a la universidad, con una variedad de trayectorias educativas y diversas expectativas. En función de las particularidades de esta población es que cuenta con una Facultad destinada a los inicios, el Instituto de Estudios Iniciales (IEI). El mismo se encarga de realizar el Curso de Preparación Universitaria que es de carácter obligatorio y no restrictivo, y también un Ciclo Inicial destinado a estudiantes que cursan en la universidad y que está formado por 4 materias: Taller de Lectura y Escritura, Problemas de la Historia Argentina, Prácticas Culturales y Matemática.

Al igual que el resto del sistema educativo, nuestra universidad se vio alterada en su ritmo normal de trabajo, a partir de la actual pandemia provocada por el COVID-19. La cuarentena obligatoria dispuesta por el gobierno nacional nos encontró en pleno transcurso del Curso de Preparación Universitaria que finalmente tuvo que ser interrumpido. No obstante, para dar continuidad pedagógica y garantizar el derecho a la educación, las autoridades de la universidad convocaron a la comunidad educativa a planificar un proceso de virtualización de aquellas materias que podían hacer una oferta curricular a distancia a través de una plataforma virtual.

Por virtualizar, asumimos aquí la idea de Orozco (2013) que considera que la misma implica la articulación de al menos esas dimensiones: lo organizacional, lo comunicacional, lo tecnológico y lo pedagógico. Por ello, a la decisión organizacional le siguió la preparación y puesta al día de la tecnología que la demanda iba a requerir, actualizando y migrando a nuevos servidores que pudieran dar respuesta al volumen de información.

Por virtualizar, asumimos aquí la idea de Orozco (2013) que considera que la misma implica la articulación de al menos esas dimensiones: lo organizacional, lo comunicacional, lo tecnológico y lo pedagógico. Por ello, a la decisión organizacional le siguió la preparación y puesta al día de la tecnología que la demanda iba a requerir, actualizando y migrando a nuevos servidores que pudieran dar respuesta al volumen de información. 
Luego, a nivel pedagógico, se fueron dando diferentes pasos. En esta presentación nos vamos a enfocar en el proceso llevado adelante por el IEl, para recibir a su nutrida matrícula y preparar las aulas virtuales. Es importante destacar que en 2020 han ingresado a la UNAJ algo más de 10000 estudiantes, por lo que cada materia del IEI oferta aproximadamente alrededor de 100 comisiones a cargo de 60 docentes. Así mismo, cada una de las materias cuenta con una coordinación que es quién se encarga habitualmente de llevar adelante los materiales de cátedra, los cronogramas y de establecer las pautas para garantizar cierta homogeneidad en la cursada. Es así como, a la hora de virtualizarlas, cada coordinación ha armado un equipo de trabajo que desarrolló el aula, la secuencia de actividades y los diversos dispositivos de interacción a partir de los materiales existentes. En ese sentido, se le brinda a cada docente el aula virtual con los contenidos diagramados y la posibilidad de intercambiar con sus estudiantes a partir de los foros, donde cada profesional tendrá que diseñar su rol docente particular. De alguna manera, será interesante analizar de qué forma este rol en este contexto tan particular se podrá reconfigurar y cómo cada docente lo asumirá y desarrollará.

Es importante destacar que la UNAJ viene realizando hace años un proceso de desarrollo e investigación en relación con la educación virtual a través de las aulas extendidas que funcionaban como complemento de la presencialidad. Así mismo, el año pasado ha comenzado un proceso de virtualización vinculado a dictar una carrera en modalidad a distancia a partir de atender la demanda de un sector en particular. Sin embargo, si bien estas experiencias son los antecedentes claros de la situación actual, resulta trascendente visualizar que la experiencia que desarrollamos actualmente se enmarca en lo que llamamos la "virtualización forzada". Desde esa perspectiva desarrollamos esta investigación a partir de vislumbrar que muchas cosas cambian su sentido en esta nueva realidad.

resulta trascendente visualizar que la experiencia que desarrollamos actualmente se enmarca en lo que llamamos la "virtualización forzada"

\section{DESCRIPCIÓN DEL DESARROLLO DE LA INVESTIGACIÓN}

Embarcados en el proceso de virtualización "forzada" antes descrito, comenzamos la investigación a partir de una indagación sobre las expectativas de las y los docentes de las materias del ciclo inicial. El cuestionario fue enviado por correo electrónico, era de carácter anónimo y podía ser respondido libremente. Se estructuró en torno a una serie de cuestiones: el contexto de aislamiento, la organización familiar y la accesibilidad, su posible o no influencia en el trabajo docente 
y en el aprendizaje del estudiantado, la falta de experiencia previa con entornos virtuales y su posible impacto, las formas de trabajo colectivo como soporte de la tarea docente. Luego un espacio libre para expresar sus expectativas respecto de esta situación.

De un total de alrededor de 250 docentes que integran las materias, recibimos 83 respuestas ${ }^{1}$ que fueron analizadas cuantitativa y cualitativamente. A nivel cuantitativo, en el siguiente cuadro se presentan el resumen de los principales hallazgos.

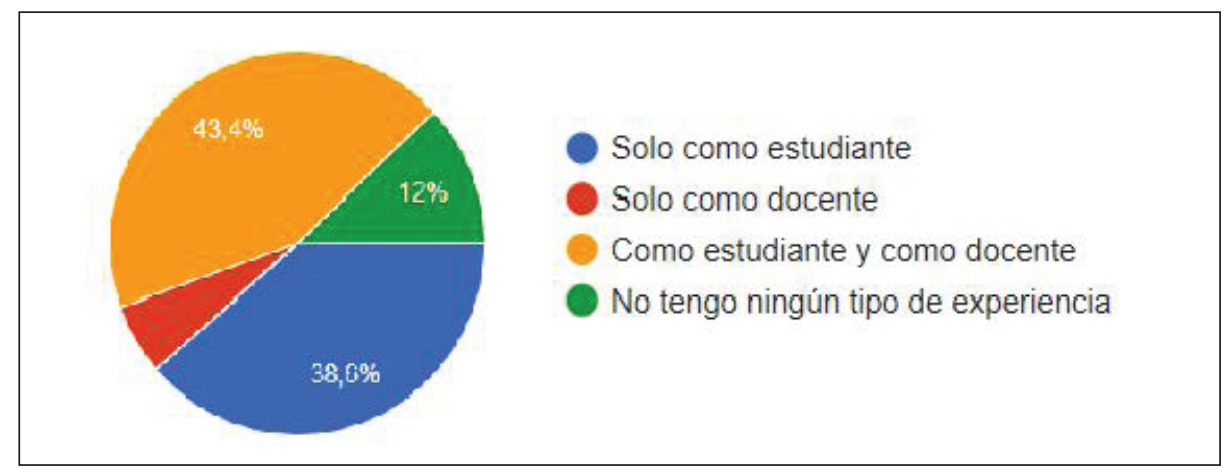

Ilustración 1 (Experiencia previa de las y los docentes con aulas virtuales)

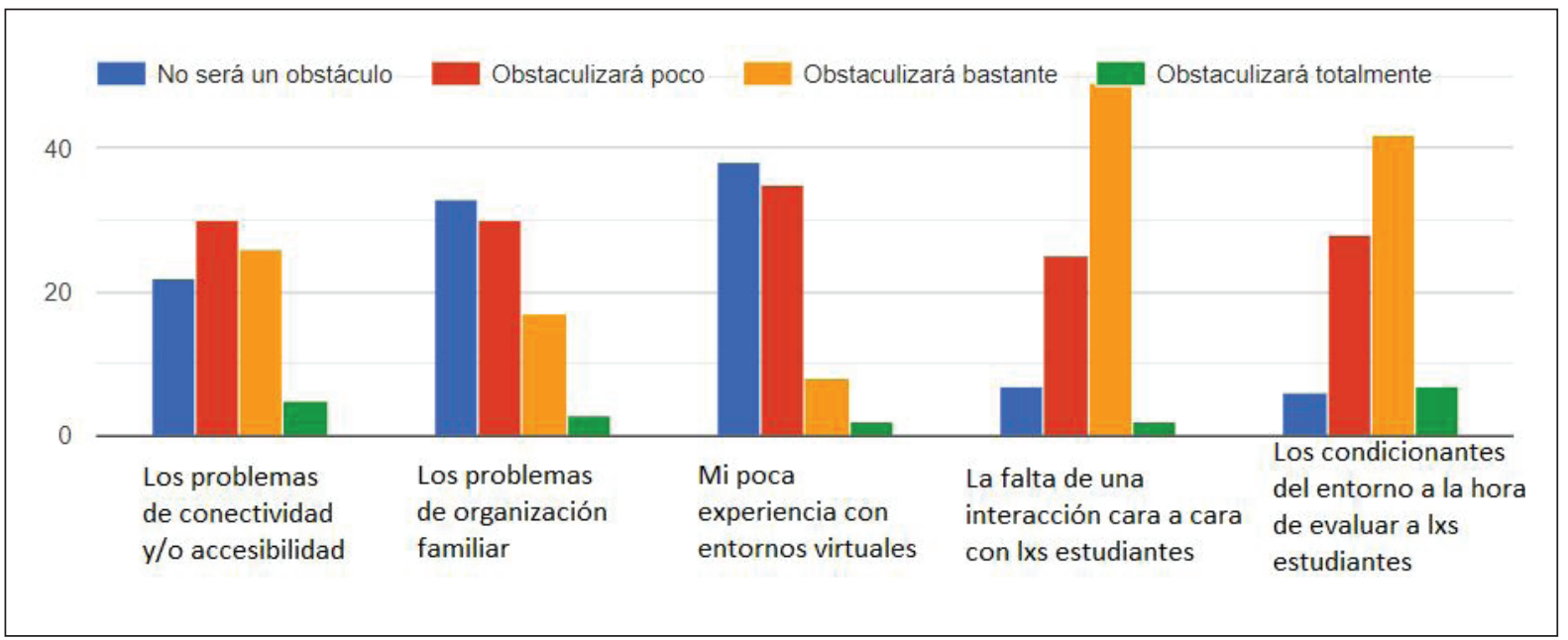

Ilustración 2 (Obstáculos a los que las y los docentes creen que tendrán que enfrentarse)

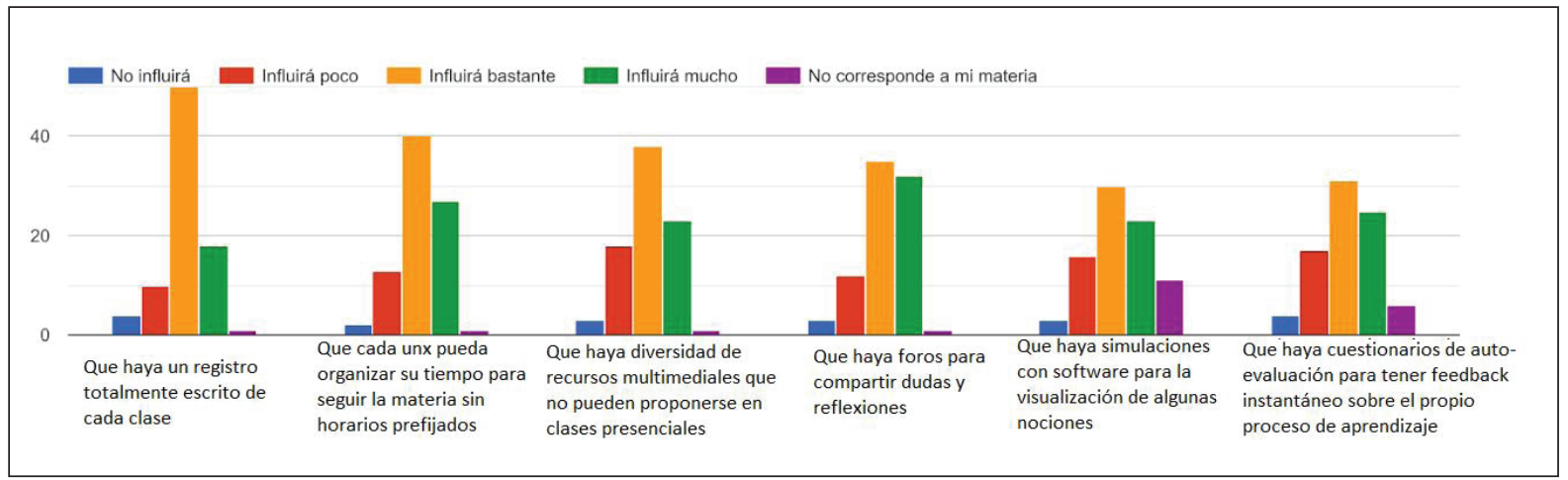

Ilustración 3 (Qué y cuánto influirá en el aprendizaje de las y los estudiantes) 
A nivel cualitativo, con la asistencia del software ATLAS.ti (versión 8.4) elaboramos diferentes esquemas y representaciones que nos permitieron entender y relacionar los principales emergentes de estas expectativas e interpretarlos en clave investigativa. Si bien la palabra más frecuente elegida por las y los docentes para caracterizar esta transición a una virtualidad forzada, fue "desafío"; diversos elementos como experiencia, recursos, clases y temporalidad, entre otros, aparecieron en las respuestas de producción breve que les propusimos para conocer tales expectativas.

Si bien la palabra más frecuente elegida por las y los docentes para caracterizar esta transición a una virtualidad forzada, fue "desafío"; diversos elementos como experiencia, recursos, clases y temporalidad, entre otros, aparecieron en las respuestas de producción breve que les propusimos para conocer tales expectativas.

\begin{tabular}{|c|c|c|c|c|c|c|c|c|c|c|}
\hline & & $\begin{array}{l}\text { Acceso } \\
\text { (1i) } 5\end{array}$ & $\begin{array}{l}\text { clase } \\
\text { (1.) } 10\end{array}$ & $\begin{array}{l}\text { Desafío } \\
\text { (iii) } 20\end{array}$ & $\begin{array}{l}\text { Distancia } \\
\text { (iii) } 5\end{array}$ & $\begin{array}{l}\text { Experiencia } \\
\text { (1i) } 33\end{array}$ & $\begin{array}{l}\text { Obstáculo } \\
\text { (1i) } 4\end{array}$ & $\begin{array}{l}\text { Recursos } \\
\text { (iii) } 17\end{array}$ & $\begin{array}{l}\text { Temor } \\
\text { (i.i. } 4\end{array}$ & $\begin{array}{l}\text { Temporalidad } \\
\text { (ii.) } 9\end{array}$ \\
\hline$\checkmark$ Acceso & (1.1) 5 & & & 3 & 1 & 1 & 3 & 1 & & \\
\hline$\nabla_{\text {clase }}$ & (1.1) 10 & & & 3 & 1 & 3 & & 2 & & 1 \\
\hline$\diamond$ Desafío & (1.) 20 & 3 & 3 & & 1 & 8 & 4 & 4 & & 3 \\
\hline$\checkmark$ Distancia & (14) 5 & 1 & 1 & 1 & & 1 & 1 & & & \\
\hline$\diamond$ Experiencia & (1.) 33 & 1 & 3 & 8 & 1 & & 1 & 7 & 2 & 4 \\
\hline$\diamond$ obstáculo & (1.1) 4 & 3 & & 4 & 1 & 1 & & 1 & & \\
\hline$\diamond$ Recursos & (11.1) 17 & 1 & 2 & 4 & & 7 & 1 & & 1 & 2 \\
\hline$\diamond$ Temor & (1.) 4 & & & & & 2 & & 1 & & \\
\hline$\checkmark$ Temporali... & (14) 9 & & 1 & 3 & & 4 & & 2 & & \\
\hline
\end{tabular}

Ilustración 4 (Tabla de coocurrencias: Las mismas son cuando dos códigos coexisten un una misma cita. El número al lado de cada código es la frecuencia de este)

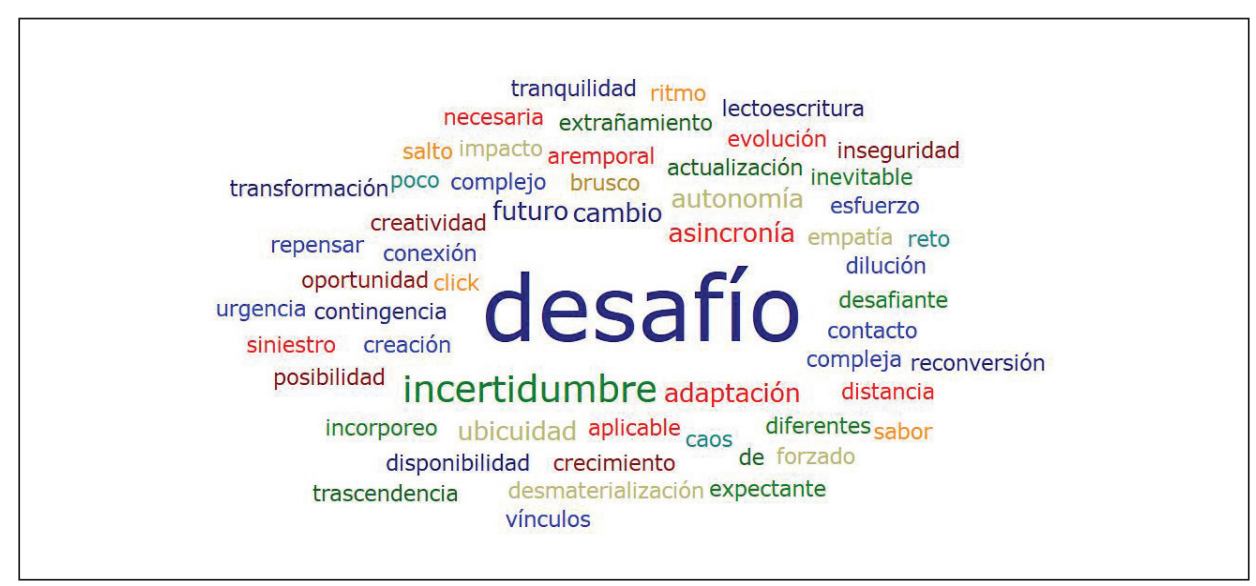

Ilustración 5 (Nube de palabras: Ofrecidas como respuesta a definir en una palabra esta etapa) 
A partir de la lectura de las respuestas de las y los docentes, comenzamos a considerar que había un supuesto que, tanto docentes como estudiantes, asumen como natural y que tenía que ver con la idea de "clase". Sin embargo, en el contexto de virtualidad forzada, para nosotras comenzaba a tener diferentes sentidos. ¿En qué consiste una clase a través de una plataforma virtual en esta situación?, ¿Quién, cómo, cuándo, cuánto explica?, ¿Cuáles son los límites espaciotemporales de la clase más allá de un cronograma de cursada? ¿Qué ofrecen las y los docentes y qué están esperando recibir las y los estudiantes en relación con los conocimientos y saberes?, son algunos de los interrogantes que nos movieron a continuar la investigación. Así, tomamos la decisión de proponernos construir el sentido de lo que se estaba entendiendo por "clase", a través de la perspectiva metodológica de la teoría fundamentada.

\section{¿En qué consiste una clase a través de una plataforma virtual en esta situación?, ¿Quién, cómo, cuándo, cuánto explica?, ¿Cuáles son los límites espaciotemporales de la clase más allá de un cronograma de cursada? ¿Qué ofrecen las y los docen- tes y qué están esperando recibir las y los estudiantes en re- lación con los conocimientos y saberes?}

Dicha metodología, desde finales de la década de los ' 60 en que tuvo sus orígenes a partir de los trabajos de Glaser \& Strauss (1967), ha ido creciendo y tomando diferentes vertientes, pasando por trabajo de revisión crítica (Glaser 1978, 2000, 2002) hasta llegar a los más recientes que han integrado aspectos relacionados con la participación y la construcción de una mirada descolonizadora (Smith 2012, Stringer 2013). En este trabajo, nosotros consideraremos los aportes en relación con la investigación educativa (Lora, Cavadias \& Miranda 2017).

Esta perspectiva metodológica asumida, se articula con aproximación teórica a la que adherimos para comprender el trabajo del docente. El enfoque documental de la didáctica (Gueudet \& Trouche 2009) resulta un marco fecundo para analizar el trabajo de quien enseña en torno a los recursos de enseñanza. Aquí se considera al término recurso en el sentido dado por Adler (2000) que contempla tanto aspectos materiales como socioculturales. Un libro de texto es un recurso, pero un intercambio con un estudiante o con un colega, una reflexión sobre la práctica, también lo son. Los recursos, según el sentido etimológico del término, "vuelven a la fuente" y alimentan el trabajo docente y potencian su desarrollo como profesional. Un recurso, forma parte en realidad de un sistema de recursos que está relacionado con el sistema de conocimientos, saberes y prácticas que el docente posee y cuya evolución en el tiempo permiten considerar este desarrollo profesional.

En el ciclo inicial de UNAJ, todas las materias tienen un libro de cátedra o recurso institucional de referencia. Si bien hemos señalado que 
la virtualización supone un entramado complejo que es mucho más amplio que transformar un libro en papel en un soporte digital, todas las materias lo han considerado como recurso central a la hora de organizar la virtualización, integrando y articulando con otros recursos multimediales que la plataforma virtual ofrece. Como sostiene el enfoque documental, el sistema de recursos es más o menos estable, y un nuevo recurso que se ofrece para su integración al sistema, implica una cierta desestabilización y reorganización de estos y por tanto una evolución. Asumimos la hipótesis de que todo el proceso de virtualización y el cambio de soporte pueden ser considerados como un nuevo recurso que las y los docentes deberán tratar de integrar a su sistema. Este proceso pondrá de manifiesto, saberes, conocimientos y prácticas en las y los docentes y, por supuesto, nuevos aprendizajes.

Este trabajo documental revela que la actividad docente va más allá de la clase, y dado la complejidad de poder definir en este nuevo contexto, en qué consiste una clase, hemos decidido avanzar en la investigación con quienes se han mostrado dispuestos a ello. A partir de la siguiente propuesta enviada por correo electrónico, que básicamente consiste en pedirle a las y los docentes que capturen una serie de pantallas que a su entender representen su trabajo asociado a una clase del tema que quisieran y que, luego, elaboren un texto o graben un audio donde puedan dar cuenta de porqué eligieron estas pantallas para representar este trabajo.

Los primeros aportes recibidos nos permiten, como parte de la fase preliminar de muestreo previsto en la metodología de la teoría fundamentada, comenzar a generar los códigos o categorías con una primera serie de propiedades, cuya matriz de síntesis presentamos a continuación:

\begin{tabular}{|l|l|}
\hline Categoría & \multicolumn{1}{c|}{ Propiedades } \\
\hline Clase & $\begin{array}{l}\text { - Temporalidad: limites; inicio/fin. } \\
\text { - Explicaciones: ¿cuándo?, ¿quién?, ¿cómo? y ¿para qué? }\end{array}$ \\
\hline Estudiantes & $\begin{array}{l}\text { - Interacciones: entre pares/con docente. } \\
\text { - Interacciones: dentro y fuera de la plataforma. } \\
\text { - Aprendizajes: ¿cómo se registran?, ¿cómo se evalúan? }\end{array}$ \\
\hline Recursos & $\begin{array}{l}\text { - Tipos y usos de los propuestos por la coordinación. } \\
\text { - Tipos y usos de los ofrecidos por la plataforma. } \\
\text { - Tipos y usos de su propia producción }\end{array}$ \\
\hline
\end{tabular}

Este primer muestreo, en la fase posterior de la investigación configurado por el contraste, necesita ser ahondado y de alguna manera servirá para refinar los códigos hermenéuticos y poder comenzar a construir la teorización sobre el sentido y significado conferido al concepto de clase virtual bajo las condiciones de virtualidad forzada descriptas oportunamente. 


\section{CONCLUSIONES}

Evidentemente dadas la fase en que nos encontramos en relación con el desarrollo de la investigación, podría conducirnos a sacar conclusiones prematuras al respecto. Esperamos que en el desarrollo de las entrevistas en profundidad proyectadas podamos construir respuestas sustantivas a los interrogantes iniciales. No obstante, creemos que las perspectivas que se abren a partir de esta pesquisa trascienden la coyuntura de la virtualidad "forzada" y nos permitirán pensar a la vez, en el día después, en la vuelta a la "presencialidad". ¿Qué de todos estos aprendizajes quedarán y se integrarán al cotidiano de nuestras clases? ¿Cómo se reconfiguran el rol docente y el rol estudiante? ¿Qué aspectos de experiencia por la virtualidad transitada podrán ser integrados y cómo en un futuro? ¿En qué medida la vuelta a la presencialidad tendrá algo de bi-modalidad aprovechando el potencial y la diversidad de recursos producidos y disponibles?, son algunas de las nuevas preguntas que nos permiten no solamente revalorizar el sentido de esta investigación circunstancial, sino proyectar posibles continuidades. 


\section{BIBLIOGRAFIA}

Adler, J. (2000). "Conceptualising resources as a theme for teacher education". Journal of Mathematics teacher Education, $n^{\circ} 3$, p. 205-224.

Glaser B. \& Strauss A. (1967). "The discovery of grounded theory: Strategies for qualitative research". New Brunswick, NJ: Aldine Transaction.

Glaser B. (1978). "Theoretical sensitivity: advances in the methodology of grounded theory", Mill Valley, Sociology Press.

Glaser B. (2000). "The discovery of the grounded theory", Mill Valley, Sociology Press.

Glaser B. (2002). “Conceptualization: On theory and theorizing using grounded theory". International Journal of Qualitative Methods, 1 (2). Article 3. Disponible en https://journals.library.ualberta.ca/ijqm/index.php/IJQM/article/view/4605

Gueudet, G. \& Trouche, L. (2009). “Towards new documentation systems for mathematics teachers?" Educational Studies in Mathematics, 71(3), 199-218.

Lora A.A., Cavadias, L. M. \& Miranda, A. J. (2017). “La Teoría Fundamentada en el Marco de la Investigación". Educativa. Saber, ciencia y libertad, 12(1), 10.

Orozco, J. (2013). “La Virtualidad en Educación Superior: Una Perspectiva. XIV Encuentro Internacional Virtual". Educa Colombia 2013. Recuperado de: http://www.virtualeduca.info/ponencias2013/171/LavirtualidadenESUnaperspectivaJairoL.pdf

Smith, L. T. (2012). "Decolonizing methodologies: Research and indigenous peoples". London, United Kingdom: Zed Books.

Stringer, E. (2013). "Action Research". Thousand Oaks, CA: SAGE. 